\title{
On inf-sup stabilized finite element methods for transient problems
}

\author{
Pavel B. Bochev ${ }^{\mathrm{a}, *, 1}$, Max D. Gunzburger ${ }^{\mathrm{b}, 2}$, John N. Shadid ${ }^{\mathrm{c}, 1}$ \\ ${ }^{a}$ Sandia National Laboratories, Computational Mathematics and Algorithms, P.O. Box 5800, MS 1110, Albuquerque, \\ NM 87185-1110, USA \\ b School of Computational Science and Information Technology, Florida State University, Tallahassee, FL 32306-4120, USA \\ ${ }^{c}$ Sandia National Laboratories, Computational Sciences, P.O. Box 5800, MS 1111, Albuquerque, NM 87185-1111, USA
}

Received 15 December 2002; received in revised form 11 September 2003; accepted 9 December 2003

\begin{abstract}
We consider the behavior of inf-sup stabilization in the context of transient problems with multiple time scales. Our motivation for studying this setting is provided by reacting flows problems for which small time steps are necessary in the integration process. We show that for algorithms defined through a process wherein spatial and temporal discretizations are separated, the coupling of implicit time integration with spatial inf-sup stabilization may lead to anomalous pressure behavior, including the onset of spurious oscillations, for very small time steps. Effectively, this coupling introduces a stability criterion resulting in a dependence between the spatial grid size and the time step. We illustrate our theoretical results by numerical examples that demonstrate the stability criterion.
\end{abstract}

(C) 2004 Elsevier B.V. All rights reserved.

Keywords: Time-dependent Stokes equations; Weak form; Coercivity; Stabilized finite element methods; Multiple time scales

\section{Introduction}

Finite element stabilization is a regularization technique that, in steady-state settings, is commonly applied in three situations:

- advection dominated problems where stabilization is applied to effect upwinding and to suppress spurious oscillations; see $[11,13,21,25]$;

\footnotetext{
${ }^{*}$ Corresponding author.

E-mail addresses: pbboche@sandia.gov (P.B. Bochev), gunzburg@csit.fsu.edu (M.D. Gunzburger), jnshadi@sandia.gov (J.N. Shadid).

${ }^{1}$ Sandia is a multiprogram laboratory operated by Sandia Corporation, a Lockheed-Martin Company, for the United States Department of Energy's National Nuclear Security Administration under contract DE-AC-94AL85000. This work was partially funded by the Applied Mathematical Sciences program, US Department of Energy, Office of Energy Research.

${ }^{2}$ Supported in part by CSRI, Sandia National Laboratories, under contract 18407.
} 
- saddle-point type weak formulations where stabilization is applied to circumvent compatibility (inf-sup) conditions by relaxing the underlying constraint; see [10,12,23,24];

- a combination of the above where stabilization serves the dual purpose of avoiding inf-sup conditions and providing the upwinding necessary for the advective terms; see [2,4-6,14,29-31].

Representative linear steady-state model problems for these three settings are the advection-diffusion problem: ${ }^{3}$

$$
-\epsilon \Delta \phi+\mathbf{b} \cdot \nabla \phi=f \quad \text { in } \Omega \text { and } \phi=0 \text { on } \Gamma,
$$

the incompressible Stokes problem:

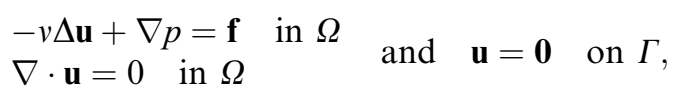

and the incompressible Oseen-type problem:

$$
\begin{aligned}
& -v \Delta \mathbf{u}+\mathbf{b} \cdot \nabla \mathbf{u}+\nabla p=\mathbf{f} \text { in } \Omega \quad \text { and } \quad \mathbf{u}=\mathbf{0} \text { on } \Gamma . \\
& \nabla \cdot \mathbf{u}=0 \quad \text { in } \Omega
\end{aligned}
$$

The formulation and analysis of stabilized methods for these steady-state problems is well understood. However, the design of robust and efficient stabilized methods for their transient counterparts is hardly a settled matter. It is generally agreed that for advection dominated problems, such as (1), stabilized methods should be based on time-space elements; see [29,30]. The original argument used to motivate application of time-space elements (see [11]) is that the time derivative and the advective term can be combined into a single "material derivative" term. Then, the extension of a method such as SUPG [21] to the transient case naturally leads to a weighting function involving this material derivative and thus the need to allow for time variation in the finite element space.

On the other hand, the need for time-space elements is not obvious at all when stabilization is applied to circumvent the inf-sup stability condition in the Stokes problem. This condition is caused by the saddlepoint nature of the mixed Galerkin formulation, namely, by the purely spatial divergence free constraint on the velocity field; see [9]. To circumvent the inf-sup condition it suffices to relax this spatial constraint which can be done without appealing to time-space elements. For the transient version of (3), the situation is even more ambiguous as now stabilization targets two different goals. If (3) is advection dominated, then time-space elements can be easily justified. But for low to moderate values of the Reynolds number, stabilization is needed only for the spatial inf-sup condition.

Some of the most effective algorithms for treating time-dependent problems can be defined through a process wherein the spatial and temporal discretizations are separated. Such algorithms are especially well adapted to the cylindrical nature of the time-space domain and usually posses excellent stability characteristics. Another reason for their popularity is that they reduce the PDE to either a system of ordinary differential equations (ODE's) or, for problems with constraints such as (2) and (3), to a system of differential algebraic equations (DAE's). In both cases the ensuing ODE or DAE system can be solved by many of the available time integration algorithms and solvers. Thus, in practice for all three cases and for several reasons, implicit, fully discrete formulations in which spatial and temporal discretizations are effected separately are in much more common use than are coupled time-space formulations. An additional

\footnotetext{
${ }^{3}$ A fourth, separate stabilization setting arises when the reaction-diffusion equation $-\epsilon \Delta \phi+\gamma \phi=f \quad$ in $\Omega$ and $\phi=0$ on $\Gamma$
} is dominated by the reaction term; see [15-17], or [26]. Because of its specifics, we do not consider this type of stabilization in our study. 
motivation for this choice is the desire to avoid the increase in the number of unknowns required to achieve more accurate in time space-time formulations.

As numerical experiences have borne out, separated, fully discrete algorithms are completely adequate for transient calculations carried out for moderate to relatively large time steps. However, in settings that require very fine time resolution, the behavior of such algorithms is not very well understood. Pollution by spurious oscillations at small time steps for parabolic problems was reported in [18] and further analyzed in [19]. For time integration schemes coupled with stabilized finite element discretization in space, instabilities have been observed for cases where a flow solver is combined with multiple time scale physics simulations, e.g., reacting flows, that require a very small time step to resolve the chemistry [28]. Instabilities were also noticed in time step convergence studies (as the time step becomes small compared to the spatial grid size) in the context of inf-sup and upwind stabilized schemes; see [2,3,28].

The issue of small time step instabilities in implicit time integration is only now beginning to attract attention from researchers and practitioners. The main reason is that most applications of implicit time integrators aim to increase rather than to decrease the time step. However, in some special circumstances when a detailed analysis of strongly coupled continuum transport and chemical reaction physics with multiple time and length scales is required, small time steps become necessary. This situation arises in engineering and scientific applications that are characterized by the co-existence of transport time scales (advection and diffusion) and very short time scales for non-equilibrium chemical reactions. The stable and accurate finite element modeling of the reaction phase may require time steps which are orders of magnitude smaller than those normally required in the flow solver. For this reason in a typical reacting flow application an implicit time stepping scheme is often preferred to allow efficient and accurate treatment of this stiff behavior.

Recent research has focused on small time step instabilities for scalar parabolic problems [18] and SUPG formulations for one-dimensional advection problems [8]. Using Rothe's method, it was shown in $[18,19]$ that implicit time discretization at sufficiently small time steps leads to singularly perturbed elliptic problems which can give rise to spurious oscillations. Bradford et al. [8] demonstrate amplification of under/ overshoots when Petrov-Galerkin spatial discretization is coupled with Crank-Nicholson implicit time integration, and the CFL number is less than one. They use a heuristic argument based on a modified equation in one space dimension to argue that small time steps lead to antidissipative effects in spatially stabilized equations. A heuristic argument is also used in [2] to motivate the addition of a spatial basis function scaled by the time step to a combined saddle-point/advection stabilized setting.

In this paper, we focus on theoretical and computational studies of small time-step instabilities when implicit, finite difference time integration is applied in conjunction with inf-sup stabilization with respect to space. With the exception of the preliminary report [7], this setting has not been studied theoretically or computationally. Another important aspect that differentiates this setting from cases considered in $[8,18]$ is that it leads to semidiscrete problems that are DAE's rather than ODE's. Here, we extend the results of [7] to the generalized trapezoidal rule and to three classes of stabilized methods for the time-dependent Stokes problem. Our interest in this setting is motivated by, among other things, the fact that it is an example of a problem for which time-space element discretizations are not easily justifiable and so one is naturally led to separate discretizations in space and time. The addition of consistent spatial stabilization then leads to additional couplings between the spatial and temporal ingredients that is not present in unstabilized mixed methods.

The paper is organized as follows. A brief summary of the notations used throughout the paper concludes this section. Section 2 is the core of the paper. There we develop the fully discrete spatially stabilized equations for the transient Stokes problem and establish sufficient coercivity conditions for the associated bilinear forms. We use these conditions in conjunction with two alternative definitions of the stability parameter that appears in stabilized methods. Our numerical results are collected in Section 3. In Section 4, a brief summary of the results concludes the paper. 


\subsection{Nomenclature}

In what follows, $\Omega$ denotes a simply connected bounded region in $\mathbb{R}^{m}, m=2,3$, with a sufficiently smooth boundary $\Gamma$ and $\mathbf{T}=(0, T)$ denotes a given time interval. Throughout the paper we employ the usual notation $H^{d}(\Omega),\|\cdot\|_{d},(\cdot, \cdot)_{d}, d \geqslant 0$, for the Sobolev spaces of all functions having square integrable derivatives up to order $d$ on $\Omega$, and the standard Sobolev norm and inner product, respectively. When $d=0$ we will write $L^{2}(\Omega)$ instead of $H^{0}(\Omega)$ and drop the index from the inner product designation. As usual, $H_{0}^{d}(\Omega)$ will denote the closure of $C_{0}^{\infty}(\Omega)$ with respect to the norm $\|\cdot\|_{d}$. Spaces consisting of vector-valued functions will be denoted in bold face.

The symbol $S_{d}^{h}$ will stand for a space of continuous, piecewise polynomial functions defined with respect to a regular partition $\mathscr{T}_{h}$; see [27], of the domain $\Omega$ into finite elements $\mathscr{K}$. For instance, $\mathscr{K}$ can be a hexahedral or a tetrahedron in three dimensions, or a triangle or a quadrilateral in two dimensions. For regular partitions finite element functions satisfy various inverse inequalities; see [27]. The two that will be used here are

$$
\left\|\Delta u_{h}\right\|_{0, \mathscr{K}} \leqslant C_{I} h^{-1}\left\|\nabla u_{h}\right\|_{0, \mathscr{K}} \quad \text { and } \quad\left\|\nabla u_{h}\right\|_{0, \mathscr{K}} \leqslant C_{I} h^{-1}\left\|u_{h}\right\|_{0, \mathscr{K}}
$$

\section{The time-dependent Stokes problem}

The main focus of this paper will be on the time-dependent, incompressible Stokes equations

$$
\begin{aligned}
& \frac{\partial \mathbf{u}}{\partial t}-\Delta \mathbf{u}+\nabla p=\mathbf{f} \quad \text { in } \Omega \times \mathbf{T}, \\
& \nabla \cdot \mathbf{u}=0 \quad \text { in } \Omega \times \mathbf{T}, \\
& \mathbf{u}=\mathbf{0} \quad \text { on } \Gamma \times \mathbf{T}, \\
& \mathbf{u}(\mathbf{x}, 0)=\mathbf{u}_{0}(\mathbf{x}) \quad \text { in } \Omega,
\end{aligned}
$$

and on fully discrete solution methods for (5)-(8) that result from the application of inf-sup stabilized discretization in space with implicit integration in time. In this equation the pressure has been scaled by the density $\rho$.

We now briefly review spatial inf-sup stabilization techniques for the steady-state problem (2). To define a mixed method for (2) we choose subspaces $\mathbf{V}^{h} \subset \mathbf{H}_{0}^{1}(\Omega)$ and $P^{h} \subset L_{0}^{2}(\Omega)$ and seek $\mathbf{u}_{h} \in \mathbf{V}^{h}, p_{h} \in P^{h}$ such that

$$
G\left(\left\{\mathbf{u}_{h}, p_{h}\right\},\left\{\mathbf{v}_{h}, \gamma q_{h}\right\}\right)=\left(\mathbf{f}, \mathbf{v}_{h}\right) \quad \forall\left(\mathbf{v}_{h}, q_{h}\right) \in\left(\mathbf{V}^{h}, P^{h}\right),
$$

where

$$
G\left(\left\{\mathbf{u}_{h}, p_{h}\right\},\left\{\mathbf{v}_{h}, \gamma q_{h}\right\}\right)=\left(\nabla \mathbf{u}_{h}, \nabla \mathbf{v}_{h}\right)-\left(p_{h}, \nabla \cdot \mathbf{v}_{h}\right)-\left(\gamma q_{h}, \nabla \cdot \mathbf{u}_{h}\right)
$$

and $\gamma= \pm 1$. We recall that (9) is not stable unless the pair $\left(\mathbf{V}^{h}, P^{h}\right)$ satisfies the inf-sup condition. Ultimately, stabilization is applied to (9) in order to circumvent this condition because it imposes significant restrictions on the choice of velocity and pressure finite elements. Since the main motivation for stabilized methods is to allow the use of equal order interpolation, throughout this paper we restrict attention to continuous pressure approximations and use the equivalent form

$$
G\left(\left\{\mathbf{u}_{h}, p_{h}\right\},\left\{\mathbf{v}_{h}, \gamma q_{h}\right\}\right)=\left(\nabla \mathbf{u}_{h}, \nabla \mathbf{v}_{h}\right)+\left(\nabla p_{h}, \mathbf{v}_{h}\right)+\left(\gamma \nabla q_{h}, \mathbf{u}_{h}\right)
$$


instead of (10). To stabilize (11), we choose a pair of weighting functions $W_{m}\left(\mathbf{v}_{h}, q_{h}\right), W_{c}\left(\mathbf{v}_{h}, q_{h}\right)$; a pair of discrete inner products $\langle\cdot, \cdot\rangle_{m},\langle\cdot, \cdot\rangle_{c}$; and seek $\mathbf{u}_{h} \in \mathbf{V}^{h}, p_{h} \in P^{h}$ such that

$$
G\left(\left\{\mathbf{u}_{h}, p_{h}\right\},\left\{\mathbf{v}_{h}, q_{h}\right\}\right)+\left\langle R_{m}\left(\mathbf{u}_{h}, p_{h}\right), W_{m}\left(\mathbf{v}_{h}, q_{h}\right)\right\rangle_{m}+\left\langle R_{c}\left(\mathbf{u}_{h}, p_{h}\right), W_{c}\left(\mathbf{v}_{h}, q_{h}\right)\right\rangle_{c}=\left(\mathbf{f}, \mathbf{v}_{h}\right)
$$

for all $\mathbf{v}_{h} \in \mathbf{V}^{h}$ and $q_{h} \in P^{h}$, where

$$
\left(\begin{array}{l}
R_{m}\left(\mathbf{u}_{h}, p_{h}\right) \\
R_{c}\left(\mathbf{u}_{h}, p_{h}\right)
\end{array}\right)=\left(\begin{array}{c}
-\Delta \mathbf{u}_{h}+\nabla p_{h}-\mathbf{f} \\
\nabla \cdot \mathbf{u}_{h}
\end{array}\right)
$$

are the residuals of (2). In what follows we focus attention to the family of standard stabilized methods. For these methods

$$
W_{c}\left(\mathbf{v}_{h}, q_{h}\right)=0 ; \quad W_{m}\left(\mathbf{v}_{h}, q_{h}\right)=\alpha \Delta \mathbf{v}_{h}-\nabla q_{h},
$$

where $\alpha$ takes the values \pm 1 or 0 , and

$$
\left\langle\mathbf{u}_{h}, \mathbf{v}_{h}\right\rangle_{m}=\sum_{\mathscr{K} \in \mathscr{T}_{h}} \tau_{\mathscr{K}}\left(\mathbf{u}_{h}, \mathbf{v}_{h}\right)_{\mathscr{K}}
$$

is a "broken" $L^{2}$ inner product weighted by a stability parameter $\tau_{\mathscr{K}}$. A standard stabilized method seeks $\mathbf{u}_{h} \in \mathbf{V}^{h}$ and $p_{h} \in P^{h}$ such that

$$
G\left(\left\{\mathbf{u}_{h}, p_{h}\right\},\left\{\mathbf{v}_{h}, \gamma q_{h}\right\}\right)-\sum_{\mathscr{K} \in \mathscr{T}_{h}} \tau_{\mathscr{K}}\left(-\Delta \mathbf{u}_{h}+\nabla p_{h}-\mathbf{f},-\alpha \Delta \mathbf{v}^{h}+\gamma \nabla q_{h}\right)_{\mathscr{K}}=\left(\mathbf{f}, \mathbf{v}_{h}\right)
$$

for all $\mathbf{v}_{h} \in \mathbf{V}^{h}, q_{h} \in P^{h}$. The method (13) is the Pressure-Poisson stabilized Galerkin [24] when $\alpha=0$; the Galerkin-Least-Squares method when $\alpha=1$ [23] and the Douglas-Wang method [12] when $\alpha=-1$. For a taxonomy of standard stabilized methods and their properties see [1]. The weight $\tau_{\mathscr{K}}$ is important ingredient of standard stabilized methods. A typical definition is

$$
\tau_{\mathscr{K}}=\delta h_{\mathscr{K}}^{2},
$$

where $h_{\mathscr{K}}$ is a measure of the element size and $\delta$ is a stabilization parameter that is independent of $h_{\mathscr{K}}$. This parameter must remain bounded away from zero because otherwise the stabilizing effect will be lost. For some methods, sufficient stability conditions also require that $\delta$ remains bounded from above; see $[10,13,23]$. Here we will assume that the proper values of this parameter are used in (13) so that the methods are stable for any conforming choice of $\mathbf{V}^{h}$ and $P^{h}$. For more details about the proper range of values for $\tau_{\mathscr{K}}$ and its origins, we refer to [10,13,20,22,29,32], and the computational study in [1].

Setting $W_{c}$ to zero excludes the residual of the continuity equation from the stabilization. This term is primarily used in conjunction with the non-linear Navier-Stokes equations where it provides an additional stabilization effect needed to cope with high advection regimes; see [29]. Here, its omission is not critical for the analyses.

\subsection{Fully discrete algorithms}

Let us now turn attention to algorithms for the time-dependent problem (5)-(8) that are defined through a process wherein the spatial and temporal discretizations are separated. When finite elements are used for spatial discretization in (5)-(8) the resulting problem will not be stable unless the pair $\left(\mathbf{V}^{h}, P^{h}\right)$ employed for this purpose satisfies the inf-sup condition. As a result, if one wishes to use an unstable pair $\left(\mathbf{V}^{h}, P^{h}\right)$, spatial discretization must necessarily include some form of regularization. Our principal goal is to investigate fully discrete methods for the transient problem (5)-(8) that rely upon the same type of spatial discretization as in (13). We will refer to these algorithms as spatially stabilized fully discrete methods. For simplicity, attention will be restricted to pairs of $C^{0}$ Lagrangian spaces defined with respect to the same triangulation 
and having the same polynomial order. For instance, in three dimensions we can set $\mathbf{V}^{h}=\left[S_{d}^{h}\right]^{3} \cap \mathbf{H}_{0}^{1}(\Omega)$ and $P^{h}=S_{d}^{h} \cap L_{0}^{2}(\Omega)$.

To derive the spatially stabilized schemes we begin in the usual manner by seeking an approximate solution to (5)-(8) in the form

$$
\mathbf{u}_{h}(\mathbf{x}, t)=\sum_{i=1}^{N} \alpha_{i}(t) \xi_{h}^{i}(\mathbf{x}) \quad \text { and } \quad p_{h}(\mathbf{x}, t)=\sum_{i=1}^{M} \beta_{i}(t) \xi_{h}^{i}(\mathbf{x}),
$$

where $\left\{\xi_{h}^{i}\right\}_{i=1}^{N}$ and $\left\{\xi_{h}^{i}\right\}_{i=1}^{M}$ denote the nodal bases for $\mathbf{V}^{h}$ and $P^{h}$, respectively. After inserting this solution into a standard weak form of (5)-(8) we obtain the (unstabilized) formulation: seek $\mathbf{u}_{h}(\cdot, t) \in \mathbf{V}^{h}$ and $p_{h}(\cdot, t) \in P^{h}$ such that

$$
\begin{aligned}
& \left(\dot{\mathbf{u}}_{h}, \mathbf{v}_{h}\right)+G\left(\left\{\mathbf{u}_{h}, p_{h}\right\},\left\{\mathbf{v}_{h}, \gamma q_{h}\right\}\right)=\left(\mathbf{f}, \mathbf{v}_{h}\right), \\
& \left(\mathbf{u}_{h}(\cdot, 0), \mathbf{v}_{h}\right)=\left(\mathbf{u}_{0}, \mathbf{v}_{h}\right)
\end{aligned}
$$

for all $\mathbf{v}_{h} \in \mathbf{V}^{h}, q_{h} \in P^{h}$ and all $t \in \mathbf{T}$. Since the pair $\left(\mathbf{V}^{h}, P^{h}\right)$ does not verify the inf-sup condition, the form $G(\cdot, \cdot)$ in (15) and (16) is unstable. To stabilize (15) and (16) spatially it would suffice to modify $G(\cdot, \cdot)$ by adding the same terms as in (13). However, if $\mathbf{u}$ is an unsteady solution of (15) and (16),

$$
-\Delta \mathbf{u}+\nabla p-\mathbf{f}=-\dot{\mathbf{u}} \neq 0
$$

and so the modified equation will not be consistent anymore. This problem can be solved by simply changing the stabilization term to

$$
-\sum_{\mathscr{K} \in \mathscr{T}_{h}} \tau_{\mathscr{K}}\left(\mathbf{u}_{h}-\Delta \mathbf{u}_{h}+\nabla p_{h}-\mathbf{f},-\alpha \Delta \mathbf{v}^{h}+\gamma \nabla q_{h}\right)_{\mathscr{K}} .
$$

The modified problem: seek $\mathbf{u}_{h}(\cdot, t) \in \mathbf{V}^{h}$ and $p_{h}(\cdot, t) \in P^{h}$ such that

$$
\begin{aligned}
& \left(\dot{\mathbf{u}}_{h}, \mathbf{v}_{h}\right)-\sum_{\mathscr{K} \in \mathscr{T}_{h}} \tau_{\mathscr{K}}\left(\dot{\mathbf{u}}_{h},-\alpha \Delta \mathbf{v}_{h}+\gamma \nabla q_{h}\right)_{\mathscr{K}}+G\left(\left\{\mathbf{u}_{h}, p_{h}\right\},\left\{\mathbf{v}_{h}, \gamma q_{h}\right\}\right) \\
& \quad-\sum_{\mathscr{K} \in \mathscr{T}_{h}} \tau_{\mathscr{K}}\left(-\Delta \mathbf{u}_{h}+\nabla p_{h},-\alpha \Delta \mathbf{v}_{h}+\gamma \nabla q_{h}\right)_{\mathscr{K}}=\left(\mathbf{f}, \mathbf{v}_{h}\right)-\sum_{\mathscr{K} \in \mathscr{T}_{h}} \tau_{\mathscr{K}}\left(\mathbf{f},-\alpha \Delta \mathbf{v}_{h}+\gamma \nabla q_{h}\right)_{\mathscr{K}}, \\
& \left(\mathbf{u}_{h}(\cdot, 0), \mathbf{v}_{h}\right)=\left(\mathbf{u}_{0}, \mathbf{v}_{h}\right)
\end{aligned}
$$

for all $\mathbf{v}_{h} \in \mathbf{V}^{h}, q_{h} \in P^{h}$ and all $t \in \mathbf{T}$ is consistent and stable.

Compared to the standard Galerkin semidiscrete equation (15) the spatially stabilized formulation has two additional terms. The role of

$$
-\sum_{\mathscr{K} \in \mathscr{T}_{h}} \tau_{\mathscr{K}}\left(-\Delta \mathbf{u}_{h}+\nabla p_{h},-\alpha \Delta \mathbf{v}_{h}+\gamma \nabla q_{h}\right)_{\mathscr{K}}
$$

is to stabilize the spatial discretization by relaxing the incompressibility constraint (6), while

$$
-\sum_{\mathscr{K} \in \mathscr{T}_{h}} \tau_{\mathscr{K}}\left(\dot{\mathbf{u}}_{h},-\alpha \Delta \mathbf{v}_{h}+\gamma \nabla q_{h}\right)_{\mathscr{K}}
$$

is required to preserve the consistency. It is easy to see that (17) and (18) is an index one system of differential algebraic equations (DAE's)

$$
\left(\begin{array}{c}
\mathbf{M} \dot{\mathbf{c}}+\underset{\mathbf{A c}}{\mathbf{A}}+\mathbf{B d} \\
\mathbf{B}^{\mathrm{T}} \mathbf{c}
\end{array}\right)+\left(\begin{array}{c}
\mathbf{R}^{\mathrm{u}} \dot{\mathbf{c}}+\mathbf{C}^{\mathrm{u}} \mathbf{c}+\mathbf{D}^{\mathrm{u}} \mathbf{d} \\
\mathbf{M}^{p} \dot{\mathbf{c}}+\mathbf{C}^{p} \mathbf{c}+\mathbf{D}^{p} \mathbf{d}
\end{array}\right)=\left(\begin{array}{l}
\mathbf{f} \\
0
\end{array}\right)+\left(\begin{array}{c}
\mathbf{f}^{\mathrm{u}} \\
\mathbf{f}^{p}
\end{array}\right)
$$


with initial condition

$$
\mathbf{c}(0)=\mathbf{c}_{0}
$$

for the unknown coefficients $\mathbf{c}=\left(\alpha_{1}(t), \ldots, \alpha_{N}(t)\right)$ and $\mathbf{d}=\left(\beta_{1}(t), \ldots, \beta_{M}(t)\right)$ of $\mathbf{u}_{h}(\mathbf{x}, t)$ and $p_{h}(\mathbf{x}, t)$, respectively. $\mathbf{M}, \mathbf{A}$ and $\mathbf{B}$ are defined in the usual manner from (15) and represent the (consistent) mass, stiffness, and gradient matrices; $\mathbf{f}$ is the Galerkin source term;

$$
\mathbf{f}^{\mathbf{u}}=-\sum_{\mathscr{K} \in \mathscr{T}_{h}} \tau_{\mathscr{K}}\left(\mathbf{f},-\alpha \Delta \xi_{h}^{i}\right)_{\mathscr{K}} \quad \text { and } \quad \mathbf{f}^{p}=-\sum_{\mathscr{K} \in \mathscr{T}_{h}} \tau_{\mathscr{K}}\left(\mathbf{f}, \gamma \nabla \xi_{h}^{i}\right)_{\mathscr{K}}
$$

The rest of the matrices in (21) are contributed by the stabilizing term (19) and the consistency term (20):

$$
\begin{aligned}
\mathbf{R}_{i j}^{\mathbf{u}}=-\sum_{\mathscr{K} \in \mathscr{T}_{h}} \tau_{\mathscr{K}}\left(\xi_{h}^{j},-\alpha \Delta \xi_{h}^{i}\right)_{\mathscr{K}} ; & \mathbf{M}_{i j}^{p}(i, j)=-\sum_{\mathscr{K} \in \mathscr{T}_{h}} \tau_{\mathscr{K}}\left(\xi_{h}^{j}, \gamma \nabla \xi_{h}^{i}\right)_{\mathscr{K}} ; \\
\mathbf{C}_{i j}^{\mathbf{u}}=-\sum_{\mathscr{K} \in \mathscr{T}_{h}} \tau_{\mathscr{K}}\left(-\Delta \xi_{h}^{j},-\alpha \Delta \xi_{h}^{i}\right)_{\mathscr{K}} ; & \mathbf{C}_{i j}^{p}=-\sum_{\mathscr{K} \in \mathscr{T}_{h}} \tau_{\mathscr{K}}\left(-\Delta \xi_{h}^{j}, \gamma \nabla \xi_{h}^{i}\right)_{\mathscr{K}} ; \\
\mathbf{D}_{i j}^{\mathbf{u}}=-\sum_{\mathscr{K} \in \mathscr{T}_{h}} \tau_{\mathscr{K}}\left(\nabla \xi_{h}^{j},-\alpha \Delta \xi_{h}\right)_{\mathscr{K}} ; & \mathbf{D}_{i j}^{p}=-\sum_{\mathscr{K} \in \mathscr{T}_{h}} \tau_{\mathscr{K}}\left(\nabla \xi_{h}^{j}, \gamma \nabla \xi_{h}^{i}\right)_{\mathscr{K}} .
\end{aligned}
$$

To write (21) more compactly let

$$
\begin{aligned}
& \mathbf{M}^{\mathbf{u}}=\mathbf{M}+\mathbf{R}^{\mathbf{u}} ; \quad \mathbf{K}^{\mathbf{u}}=\mathbf{A}+\mathbf{C}^{\mathbf{u}} ; \quad \mathbf{K}^{p}=\mathbf{B}^{\mathrm{T}}+\mathbf{C}^{p} ; \\
& \mathbf{G}^{\mathbf{u}}=\mathbf{B}+\mathbf{D}^{\mathbf{u}} ; \quad \mathbf{G}^{p}=\mathbf{D}^{p} ; \quad \mathbf{f}^{\mathbf{u}}:=\mathbf{f}+\mathbf{f}^{\mathbf{u}} ;
\end{aligned}
$$

so that (21) takes the form

$$
\left(\begin{array}{l}
\mathbf{M}^{\mathbf{u}} \dot{\mathbf{c}}+\mathbf{K}^{\mathbf{u}} \mathbf{c}+\mathbf{G}^{\mathbf{u}} \mathbf{d} \\
\mathbf{M}^{p} \dot{\mathbf{c}}+\mathbf{K}^{p} \mathbf{c}+\mathbf{G}^{p} \mathbf{d}
\end{array}\right)=\left(\begin{array}{c}
\mathbf{f}^{\mathbf{u}} \\
\mathbf{f}^{p}
\end{array}\right)
$$

The system of DAE's (23) and (22), or equivalently (17) and (18) may be solved by any of the available DAE solvers. In this paper we use the $\theta$-method, also known as the Generalized Trapezoidal Rule. To discretize in time, the interval $(0, T)$ is subdivided into $L$ subintervals $\left[t_{k}, t_{k+1}\right], k=0, \ldots, L$ with lengths $\Delta_{t}^{k}$. Throughout, $\mathbf{f}^{\mathbf{u}, k}=\mathbf{f}^{\mathbf{u}}\left(t_{k}\right), \mathbf{f}^{p, k}=\mathbf{f}^{p}\left(t_{k}\right) ; \mathbf{u}_{h}^{k}, p_{h}^{k}, \mathbf{c}^{k}$ and $\mathbf{d}^{k}$ denote approximations to $\mathbf{u}_{h}\left(\mathbf{x}, t_{k}\right), p_{h}\left(\mathbf{x}, t_{k}\right), \mathbf{c}\left(t_{k}\right)$ and $\mathbf{d}\left(t_{k}\right)$, respectively. Given $\mathbf{c}^{0}, \mathbf{c}^{k+1}$ and $\mathbf{d}^{k+1}$ for $k=0,1, \ldots, L-1$ are determined from:

$$
\frac{1}{\Delta_{t}^{k}}\left(\begin{array}{c}
\mathbf{M}^{\mathbf{u}}\left(\mathbf{c}^{k+1}-\mathbf{c}^{k}\right) \\
\mathbf{M}^{p}\left(\mathbf{c}^{k+1}-\mathbf{c}^{k}\right)
\end{array}\right)=\left(\begin{array}{c}
\mathbf{f}_{\theta}^{\mathbf{u}, k} \\
\mathbf{f}_{\theta}^{p, k}
\end{array}\right)-\left(\begin{array}{c}
\mathbf{K}^{\mathbf{u}} \mathbf{c}_{\theta}^{k}+\mathbf{G}^{\mathbf{u}} \mathbf{d}_{\theta}^{k} \\
\mathbf{K}^{p} \mathbf{c}_{\theta}^{k}+\mathbf{G}^{p} \mathbf{d}_{\theta}^{k}
\end{array}\right),
$$

where

$$
\mathbf{c}_{\theta}^{k}=\theta \mathbf{c}^{k+1}+(1-\theta) \mathbf{c}^{k} \quad \text { and } \quad \mathbf{d}_{\theta}^{k}=\theta \mathbf{d}^{k+1}+(1-\theta) \mathbf{d}^{k}
$$

and likewise for $\mathbf{f}_{\theta}^{\mathbf{u}, k}$ and $\mathbf{f}_{\theta}^{p, k}$. The value of $\theta$ ranges between 0 and 1 . For $\theta=0$ the scheme (24) is the explicit Euler method, $\theta=1 / 2$ gives the second-order neutrally stable Crank-Nicholson method, and $\theta=1$ gives the first-order accurate implicit Euler rule.

Clearly, (24) is a system of linear algebraic equations

$$
\left(\begin{array}{ll}
\frac{1}{\Delta_{t}^{k}} \mathbf{M}^{\mathbf{u}}+\theta \mathbf{K}^{\mathbf{u}} & \theta \mathbf{G}^{\mathbf{u}} \\
\frac{1}{\Delta_{t}^{k}} \mathbf{M}^{p}+\theta \mathbf{K}^{p} & \theta \mathbf{G}^{p}
\end{array}\right)\left(\begin{array}{c}
\mathbf{c}^{k+1} \\
\mathbf{d}^{k+1}
\end{array}\right)=\left(\begin{array}{c}
\mathbf{f}_{\theta}^{\mathbf{u}, k} \\
\mathbf{f}_{\theta}^{p, k}
\end{array}\right)+\left(\begin{array}{cc}
\frac{1}{\Delta_{t}^{k}} \mathbf{M}^{\mathbf{u}}-(1-\theta) \mathbf{K}^{\mathbf{u}} & (1-\theta) \mathbf{G}^{\mathbf{u}} \\
\frac{1}{\Delta_{t}^{k}} \mathbf{M}^{p}-(1-\theta) \mathbf{K}^{p} & (1-\theta) \mathbf{G}^{p}
\end{array}\right)\left(\begin{array}{c}
\mathbf{c}^{k} \\
\mathbf{d}^{k}
\end{array}\right)
$$


for the unknown coefficients $\mathbf{c}^{k+1}$ and $\mathbf{d}^{k+1}$. We note that $\mathbf{M}^{\mathbf{u}}$ and $\mathbf{M}^{p}$ are not symmetric mass matrices because they include contributions from stabilizing terms. As a result, even for $\theta=0$ it may not be possible to lump these matrices and solve (25) explicitly.

Our main interest is to investigate how solutions of the spatially stabilized fully discrete method (25) behave when the spatial mesh remains fixed and $\Delta_{t}^{k} \mapsto 0$. It is not difficult to see that the matrix of the linear system in (25) is engendered by the parameter-dependent bilinear form

$$
\begin{aligned}
B\left(\left\{\mathbf{u}_{h}, p_{h}\right\},\left\{\mathbf{v}_{h}, \gamma q_{h}\right\} ; \rho\right)= & \frac{1}{\rho}\left(\left(\mathbf{u}_{h}, \mathbf{v}_{h}\right)-\sum_{\mathscr{K} \in \mathscr{T}_{h}} \tau_{\mathscr{K}}\left(\mathbf{u}_{h},-\alpha \Delta \mathbf{v}_{h}+\gamma \nabla q_{h}\right)_{\mathscr{K}}\right) \\
& +\theta\left(G\left(\left\{\mathbf{u}_{h}, p_{h}\right\},\left\{\mathbf{v}_{h}, \gamma q_{h}\right\}\right)-\sum_{\mathscr{K} \in \mathscr{T}_{h}} \tau_{\mathscr{K}}\left(-\Delta \mathbf{u}_{h}+\nabla p_{h},-\alpha \Delta \mathbf{v}_{h}+\gamma \nabla q_{h}\right)_{\mathscr{K}}\right),
\end{aligned}
$$

where $\rho$ represents the current time step. Therefore, spatial stability of the implicit time step in (25) can be inferred from the stability properties of the associated bilinear form (26). When $\rho \mapsto 0$, one potential source of problems was documented in (17). There it was noted that implicit time discretization of parabolic problems at sufficiently small time steps leads to singularly perturbed elliptic problems and a possibility for spurious oscillations in the vicinity of thin layers. The singular perturbation is due to the standard mass term $\mathbf{M}$.

In this paper we will focus on another aspect of (26) that may have a potential destabilizing effect, namely, the presence of the extra "mass" term (20), introduced in (17) to preserve consistency. This term couples the time derivative of the velocity field with the spatial weight function $W_{m}\left(\mathbf{v}_{h}, q_{h}\right)$. Unlike the true mass term, (20) is non-symmetric, indefinite and includes higher order derivatives. As a result, for small $\rho$ this term may dominate $B(\cdot, \cdot)$, leading to a loss of spatial stability.

\subsection{Stability analysis of fully discrete forms}

We will consider stability of (26) for $\gamma=-1$. Note that (26) is a weighted average of the two mass terms and the spatially stabilized mixed Galerkin form from (13). Also note that the "mass" term (20) leads to the coupling $\tau_{\mathscr{K}} / \rho$ between the spatial stability parameter and the time step. Therefore, coercivity of (26) will depend on all three parameters $\theta, \rho$ and $\tau_{\mathscr{K}}$. The next theorem quantifies this dependence and establishes a sufficient condition for maintaining stable approximations. To state the main result assume that there are two positive numbers $\tau_{1}$ and $\tau_{2}$ such that

$$
0<\tau_{1} \leqslant \tau_{\mathscr{K}} \leqslant \tau_{2} \text { for all } \mathscr{K} \in \mathscr{T}_{h} .
$$

This assumption is reasonable for regular partitions of the domain $\Omega$ into finite elements. Moreover, for uniform grids we can further assume that $\tau_{1}=\tau_{2}=\tau$.

Theorem 1. Assume that $\mathscr{T}_{h}$ is a regular triangulation and let $\mathbf{V}^{h}=\left[S_{d}^{h}\right]^{N} \cap \mathbf{H}_{0}^{1}(\Omega)$ and $P^{h}=S_{r}^{h} \cap L_{0}^{2}(\Omega)$ for some $d \geqslant 2$ and $r \geqslant 1$. Then, for ${ }^{4} 0<\theta \leqslant 1$

\footnotetext{
${ }^{4}$ We recall that temporal stability requires $1 / 2 \leqslant \theta \leqslant 1$. Here we consider spatial stability of one implicit time step and for the sake of generality $\theta$ is allowed to assume any value in the interval $(0,1]$.
} 


$$
B\left(\left\{\mathbf{u}_{h}, p_{h}\right\},\left\{\mathbf{u}_{h},-p_{h}\right\} ; \rho\right) \geqslant\left(\frac{h^{2}}{4 \rho C_{I}^{2}}+C_{1}\left(\alpha, \theta, \rho, \tau_{\mathscr{K}}\right)\right)\left\|\nabla \mathbf{u}_{h}\right\|_{0}^{2}+C_{2}\left(\alpha, \theta, \rho, \tau_{K}\right)\left\|\nabla p_{h}\right\|_{0}^{2},
$$

where for $\alpha=0$ (Pressure-Poisson stabilization):

$$
C_{1}=\theta\left(1-\frac{\tau_{2} C_{I}^{2}}{2 h^{2}}\right) \quad \text { and } \quad C_{2}=\frac{\tau_{1}}{2}\left(\theta-\frac{\tau_{2}}{\rho}\right)
$$

for $\alpha=1$ (Galerkin-Least-Squares stabilization):

$$
C_{1}=\left(\theta-\frac{\tau_{2} C_{I}^{2}}{2 h^{2}}\left(\theta+2 \tau_{2}\right)\right) \quad \text { and } \quad C_{2}=\frac{\tau_{1}}{2}\left(\theta-\frac{\tau_{2}}{2 \rho}\right),
$$

and for $\alpha=-1$ (Douglas-Wang stabilization):

$$
C_{1}=\left(\theta-\frac{\tau_{2} C_{I}^{2}}{2 h^{2}}\left(\theta(v-1)+2 \tau_{2}\right)\right) \quad \text { and } \quad C_{2}=\frac{\tau_{1}}{2}\left(\theta\left(1-\frac{1}{v}\right)-\frac{\tau_{2}}{2 \rho}\right),
$$

with $v>1$ an arbitrary real number.

Proof. Setting $\left(\mathbf{v}_{h}, q_{h}\right)$ equal to $\left(\mathbf{u}_{h},-p_{h}\right)$ in (26) gives

$$
\begin{aligned}
B\left(\left\{\mathbf{u}_{h}, p_{h}\right\},\left\{\mathbf{u}_{h},-p_{h}\right\} ; \rho\right)= & \frac{1}{\rho}\left(\left\|\mathbf{u}_{h}\right\|_{0}^{2}+\sum_{\mathscr{K} \in \mathscr{T}_{h}} \tau_{\mathscr{K}}\left(\mathbf{u}_{h}, \alpha \Delta \mathbf{u}_{h}+\nabla p_{h}\right)_{\mathscr{K}}\right) \\
& +\theta\left(\left\|\nabla \mathbf{u}_{h}\right\|_{0}^{2}+\sum_{\mathscr{K} \in \mathscr{T}_{h}} \tau_{\mathscr{K}}\left(-\Delta \mathbf{u}_{h}+\nabla p_{h}, \alpha \Delta \mathbf{u}_{h}+\nabla p_{h}\right)_{\mathscr{K}}\right) .
\end{aligned}
$$

First we bound the "mass" term originating from (20). Using Cauchy's, the $\varepsilon$ and the inverse (4) inequalities

$$
\begin{aligned}
\frac{\tau_{\mathscr{K}}}{\rho}\left(\mathbf{u}_{h}, \alpha \Delta \mathbf{u}_{h}+\nabla p_{h}\right)_{\mathscr{K}} \leqslant \frac{\tau_{\mathscr{K}}}{\rho}\left[|\alpha|\left\|\mathbf{u}_{h}\right\|_{0, \mathscr{K}}\left\|\Delta \mathbf{u}_{h}\right\|_{0, \mathscr{K}}+\left\|\mathbf{u}_{h}\right\|_{0, \mathscr{K}}\left\|\nabla p_{h}\right\|_{0, \mathscr{K}}\right] & \\
& \leqslant \begin{cases}\frac{1}{2 \rho}\left\|\mathbf{u}_{h}\right\|_{0, \mathscr{K}}^{2}+\frac{\tau_{\mathscr{K}}^{2}}{2 \rho}\left\|\nabla p_{h}\right\|_{0, \mathscr{K}}^{2} & \text { for } \alpha=0, \\
\frac{3}{4 \rho}\left\|\mathbf{u}_{h}\right\|_{0, \mathscr{K}}^{2}+\frac{\tau_{\mathscr{K}}^{2}}{2 \rho}\left\|\nabla p_{h}\right\|_{0, \mathscr{K}}^{2}+\frac{C_{I}^{2} \tau_{\mathscr{K}}^{2}}{h^{2}}\left\|\nabla \mathbf{u}_{h}\right\|_{0, \mathscr{K}}^{2} & \text { for } \alpha= \pm 1 .\end{cases}
\end{aligned}
$$

Summing the last inequality over all elements and using (27),

$$
\sum_{\mathscr{K} \in \mathscr{T}_{h}} \frac{\tau_{\mathscr{K}}}{\rho}\left(\mathbf{u}_{h}, \alpha \Delta \mathbf{u}_{h}+\nabla p_{h}\right)_{\mathscr{K}} \leqslant \begin{cases}\frac{1}{2 \rho}\left\|\mathbf{u}_{h}\right\|_{0}^{2}+\frac{\tau_{2}^{2}}{2 \rho}\left\|\nabla p_{h}\right\|_{0}^{2} & \text { for } \alpha=0, \\ \frac{3}{4 \rho}\left\|\mathbf{u}_{h}\right\|_{0}^{2}+\frac{\tau_{2}^{2}}{2 \rho}\left\|\nabla p_{h}\right\|_{0}^{2}+\frac{C_{I}^{2} \tau_{2}^{2}}{h^{2}}\left\|\nabla \mathbf{u}_{h}\right\|_{0}^{2} & \text { for } \alpha= \pm 1 .\end{cases}
$$

Next, we estimate from below the spatial stabilization term originating from (19). Using Cauchy's, $\varepsilon$ and inverse (4) inequalities

$$
\tau_{\mathscr{K}}\left(-\Delta \mathbf{u}_{h}+\nabla p_{h}, \alpha \Delta \mathbf{u}_{h}+\nabla p_{h}\right)_{\mathscr{K}} \geqslant \begin{cases}\frac{\tau_{\mathscr{K}}}{2}\left\|\nabla p_{h}\right\|_{0, \mathscr{K}}^{2}-\frac{\tau_{\mathscr{K}} C_{I}^{2}}{2 h^{2}}\left\|\nabla \mathbf{u}_{h}\right\|_{0, \mathscr{K}}^{2} & \text { for } \alpha=0, \\ \tau_{\mathscr{K}}\left\|\nabla p_{h}\right\|_{0, \mathscr{K}}^{2}-\frac{\tau_{\mathscr{K}} C_{I}^{2}}{h^{2}}\left\|\nabla \mathbf{u}_{h}\right\|_{0, \mathscr{K}}^{2} & \text { for } \alpha=1, \\ \tau_{\mathscr{K}}\left(1-\frac{1}{v}\right)\left\|\nabla p_{h}\right\|_{0, \mathscr{K}}^{2}-(v-1) \frac{\tau_{\mathscr{K}} C_{I}^{2}}{2 h^{2}}\left\|\nabla \mathbf{u}_{h}\right\|_{0, \mathscr{K}}^{2} & \text { for } \alpha=-1 .\end{cases}
$$


Summing over all elements and using (27) gives

$$
\sum_{\mathscr{K} \in \mathscr{T}_{h}} \tau_{\mathscr{K}}\left(-\Delta \mathbf{u}_{h}+\nabla p_{h}, \alpha \Delta \mathbf{u}_{h}+\nabla p_{h}\right)_{\mathscr{K}} \geqslant \begin{cases}\frac{\tau_{1}}{2}\left\|\nabla p_{h}\right\|_{0}^{2}-\frac{\tau_{2} C_{I}^{2}}{2 h^{2}}\left\|\nabla \mathbf{u}_{h}\right\|_{0}^{2} & \text { for } \alpha=0, \\ \tau_{1}\left\|\nabla p_{h}\right\|_{0}^{2}-\frac{\tau_{2} C_{I}^{2}}{h^{2}}\left\|\nabla \mathbf{u}_{h}\right\|_{0}^{2} & \text { for } \alpha=1, \\ \tau_{1}\left(1-\frac{1}{v}\right)\left\|\nabla p_{h}\right\|_{0}^{2}-(v-1) \frac{\tau_{2} C_{I}^{2}}{2 h^{2}}\left\|\nabla \mathbf{u}_{h}\right\|_{0}^{2} & \text { for } \alpha=-1,\end{cases}
$$

where $v>1$ is arbitrary real number. Combining (29) and (30) shows that

$$
B\left(\left\{\mathbf{u}_{h}, p_{h}\right\},\left\{\mathbf{u}_{h},-p_{h}\right\} ; \rho\right) \geqslant \frac{1}{4 \rho}\left\|\mathbf{u}_{h}\right\|_{0}^{2}+C_{1}\left(\alpha, \theta, \rho, \tau_{\mathscr{K}}\right)\left\|\nabla \mathbf{u}_{h}\right\|_{0}^{2}+C_{2}\left(\alpha, \theta, \rho, \tau_{K}\right)\left\|\nabla p_{h}\right\|_{0}^{2},
$$

where $C_{1}$ and $C_{2}$ are the constants from (28). The theorem now follows by using the inverse inequality (4) to bound the $L^{2}$ norm of the velocity from below by the $L^{2}$ norm of its gradient.

Theorem 1 implies that a sufficient spatial stability condition for the fully discrete problem is to require

$$
\left(\frac{h^{2}}{4 \rho C_{I}^{2}}+C_{1}\left(\alpha, \theta, \rho, \tau_{\mathscr{K}}\right)\right) \geqslant \eta_{1} \quad \text { and } \quad C_{2}\left(\alpha, \theta, \rho, \tau_{\mathscr{K}}\right) \geqslant \eta_{2}
$$

where, ideally, $\eta_{i}>0$ should be independent of $h, \rho$ and $\tau$. To see what (31) means in terms of a condition on $\tau_{\mathscr{K}}$ and $\rho$, we must first select a definition of $\tau_{\mathscr{K}}$. Among the multitude of available definitions we choose two that exemplify the two opposing avenues of approach to construction of $\tau$. To avoid unnecessary technicalities we consider a uniform triangulation $\mathscr{T}_{h}$ so that $\tau_{\mathscr{K}}$ can take the same value on each element.

The first approach is to argue that since the role of $\tau$ in the present context is to effect spatial inf-sup stabilization it should not depend on the time step and so $\tau$ should be defined according to the steady-state formula (14). For uniform triangulations (14) reduces to $\tau=\delta h^{2}$ where $\delta$ is a suitable stabilization parameter independent of $h$ (and $\rho$ ). We will refer to this choice as the spatial stability parameter, $\tau_{\mathrm{S}}$.

The second approach is to view the model equations as the limit case of a wider class of transient problems with advection. For such problems definitions of $\tau$ include a time scale variation and advective stabilization scale; see, e.g., $[4,14,32]$ and $[29,30]$ for definitions in space-time settings. The alternative to $\tau_{\mathrm{S}}$ would be to specialize a formula from the transient setting with advection by dropping the advective scale. For uniform meshes this gives the following definition:

$$
\tau=\left(\frac{1}{\rho^{2}}+\frac{1}{\delta h^{4}}\right)^{-1 / 2}=\rho\left(1+\frac{\rho^{2}}{\delta h^{4}}\right)^{-1 / 2} .
$$

Here $\delta$ is another parameter independent from $h$ and $\rho$. This choice will be referred to as the transient stability parameter, $\tau_{\mathrm{T}}$.

For $\tau_{\mathrm{S}}$, sufficient stability conditions (31) specialize to

$$
\left(\frac{h^{2}}{4 \rho C_{I}^{2}}+\theta\left(1-\frac{\delta C_{I}^{2}}{2}\right)\right)>0 \text { and } \theta-\frac{\delta h^{2}}{\rho}>0
$$

when $\alpha=0$,

$$
\left(\frac{h^{2}}{4 \rho C_{I}^{2}}+\theta-\frac{\delta C_{I}^{2}}{2}\left(\theta+2 \delta h^{2}\right)\right)>0 \quad \text { and } \quad \theta-\frac{\delta h^{2}}{2 \rho}>0
$$


when $\alpha=1$, and

$$
\left(\frac{h^{2}}{4 \rho C_{I}^{2}}+\theta-\frac{\delta C_{I}^{2}}{2}\left(\theta(v-1)+2 \delta h^{2}\right)\right)>0 \quad \text { and } \quad \theta\left(1-\frac{1}{v}\right)-\frac{\delta h^{2}}{2 \rho}>0
$$

when $\alpha=-1$. Thanks to the term $1 / 4 \rho$ the first inequality in (33)-(35) will be satisfied for a sufficiently small $\rho$. Thus, for a fixed $h$ and $\rho \mapsto 0$ we are guaranteed that the coercivity constant for the velocity norm in (28) will remain positive. Moreover, for $\rho \mapsto 0$ this constant will grow. As a result, for small $\rho$ we can expect that stability of the form with respect to the velocity field will actually improve.

However, the second stability inequality in (33)-(35) will fail unless

$$
h^{2}<C(\theta) \frac{\rho}{\delta}
$$

where $C(\theta)=\theta$ for (33), $C(\theta)=2 \theta$ for (34) and $C(\theta)=2 \theta(1-1 / v)$ for (35). This relation between $\rho$ and $h$ is reminiscent of the stability conditions for explicit time integration schemes, except that now it is the spatial step that is bounded by the time step.

It is worth pointing out that (36) has appeared in the context of other stabilized methods as well. For instance Blasco and Codina require essentially the same stability condition to prove time and space error estimates for a fully discrete stabilized method for the Navier-Stokes equations; see [5,6]. Their method, however, employs a different kind of stabilization that is motivated by the Chorin projection scheme and does not use the residual of the momentum equation. The fact that the same condition arises in different stabilizations suggests that there's a certain degree of universality in (36), or at least, that one should not dismiss it as an artifact from the analysis. Our numerical experiments will reaffirm this conclusion.

Consider next the transient definition (32). For small time steps $\tau_{\mathrm{T}}=\mathrm{O}(\rho)$ and

$$
\left(\frac{h^{2}}{4 \rho C_{I}^{2}}+C_{1}\left(\alpha, \theta, \rho, \tau_{\mathscr{K}}\right)\right)>0
$$

so that the first inequality in (33)-(35) holds. Also, from (32) we see that $\tau_{\mathrm{T}}<\rho$ for any $\delta, h$ and $\rho$. As a result, at least for $\theta=1$, we are guaranteed that $C_{2}>0$ for any value of $\rho$. However, since for small time steps $\tau_{\mathrm{T}}=\mathrm{O}(\rho)$,

$$
C_{2}=\mathrm{O}\left(\rho^{3}\right)
$$

For instance, for the Pressure-Poisson stabilized method $(\alpha=0)$

$$
\theta-\frac{\tau_{\mathrm{T}}}{\rho}=\mathrm{O}\left(\frac{\rho^{2}}{h^{4}}\right) ; \quad \text { and } \quad \frac{\tau_{\mathrm{T}}}{2}\left(\theta-\frac{\tau_{\mathrm{T}}}{\rho}\right)=\mathrm{O}\left(\rho^{3}\right)
$$

Consequently, for fixed $h$ and $\rho \mapsto 0$ the coercivity constant of the pressure term will rapidly decrease, effectively reducing (26) to an unstable mixed method. In this case it is clear that small time steps will cause problems in the solution.

For $\tau_{\mathrm{S}}$ we cannot draw the same definitive conclusion because our stability conditions are only sufficient but not necessary for the coercivity of (26). As a result, their violation may not necessarily result in an unstable form. A proper way to interpret these conditions is to treat them as cautionary warnings that very small time steps may possibly cause problems. Nevertheless, our conditions allow us to predict what will be the most likely manifestation of these problems should they actually occur. For both the spatial and the transient $\tau$ definitions the first inequality in (33)-(35) was never violated. In fact, form (26) becomes "more positive" with respect to the velocity variable as $\rho \mapsto 0$. Thus, it is unlikely that velocity approximation will suffer from serious problems at small time steps. On the other hand, the second inequality was violated for 
both $\tau$ definitions. This allows us to conclude that the most likely victim of small time steps will be the pressure approximation. Another observation that can be easily verified using (32) is that

$$
\tau_{\mathrm{T}} \approx \tau_{\mathrm{S}}
$$

whenever $\delta$ and $\rho$ satisfy condition (36). For such values, $\tau_{\mathrm{T}}$ is dominated by the spatial discretization scale and it can be expected that both definitions will give close if not identical results.

We test these conjectures numerically in the next section.

\section{Numerical results}

In this section we present numerical results that complement the theoretical analysis of Theorem 1. Our goal is to determine to what extent violation of the sufficient conditions in this theorem is reflected in numerical instabilities or other anomalies at very small time steps.

In all experiments $\Omega$ is the unit square in $\mathbf{R}^{2}$. To test numerical stability of (26) we perform one step of the implicit Euler method ((24) with $\theta=1)$, for the exact steady-state solution

$$
\begin{aligned}
& \mathbf{u}=\left(\begin{array}{c}
\sin (\pi x-0.7) \sin (\pi y+0.2) \\
\cos (\pi x-0.7) \cos (\pi y+0.2)
\end{array}\right) \\
& p(x, y)=\sin (x) \cos (y)+(\cos (1)-1) \sin (1) .
\end{aligned}
$$

The initial condition in (8) is set equal to the exact solution and $\mathbf{f}$ is computed by evaluating the momentum equation (5) for the exact solution. Then we solve (24) with $\mathbf{c}^{0}$ initialized by the finite element interpolant of the exact velocity field and

$$
\rho_{n}=10^{-n} \text { for } n=1,2,3,4,5,6 .
$$

In all experiments, the pressure and velocity are approximated by piecewise quadratic finite elements defined with respect to the same uniform triangulation of $\Omega$ into triangles. The mesh is obtained by dividing $\Omega$ into 100 squares and then drawing the diagonal in each square. All matrices are assembled using a sevenpoint quadrature rule and the linear system is solved using a direct solver. Note that an initial condition for the pressure is not required for $\theta=1$. We recall that the P2-P2 pair employed here does not satisfy the inf-sup condition.

To establish a reference point for evaluation of the possible impact from the stabilizing term (19) and the consistency term (20), we compute a mixed Galerkin finite element solution using the same mesh and the stable Taylor-Hood pair. This solution is obtained following the same procedure, i.e., by one step of the implicit Euler method, but applied to the unstabilized problem (15) and (16). Table 1 shows the $L^{2}$ errors of the velocity and pressure approximations and the $H^{1}$ error for the velocity. We observe a slight deterioration in the pressure approximation as the time step is being decreased. Pressure contours in Fig. 1 show the onset of spurious oscillations for the smallest time step that can be expected to occur according to the analyses of [18].

We now turn attention to the three stabilized methods. Results are presented for spatial and transient stability parameters. Both definitions require a choice of the real parameter $\delta$. For each fully discrete algorithm we chose the value that minimized the error in the companion steady-state method for the given mesh. For the Pressure-Poisson and Douglas-Wang methods this value turned out to be $\delta=0.05$. For the Galerkin-Least-Squares we found that the smallest error occurs at $\delta=0.04$. Tables $2-4$ list the $L^{2}$ and $H^{1}$ errors for the six different time steps. For comparison, the third row in each table gives the errors computed by the associated steady-state stabilized method. 
Table 1

Finite element errors after one implicit Euler step: Taylor-Hood spatial discretization

\begin{tabular}{llll}
\hline$n$ & Velocity & \multicolumn{2}{c}{$\begin{array}{l}\text { Pressure } \\
L^{2}\end{array}$} \\
\cline { 2 - 4 } & $L^{2}$ & $H^{1}$ & $0.67770 \mathrm{D}-03$ \\
$1>0.0005$ & $0.39334 \mathrm{D}-03$ & $0.30349 \mathrm{D}-01$ & $0.69915 \mathrm{D}-03$ \\
2 & $0.39244 \mathrm{D}-03$ & $0.30349 \mathrm{D}-01$ & $0.90321 \mathrm{D}-03$ \\
3 & $0.39239 \mathrm{D}-03$ & $0.30352 \mathrm{D}-01$ & $0.15369 \mathrm{D}-02$ \\
$\rho<0.0005$ & & & $0.18965 \mathrm{D}-02$ \\
4 & $0.39477 \mathrm{D}-03$ & $0.30390 \mathrm{D}-01$ & $0.66562 \mathrm{D}-01$ \\
\hline
\end{tabular}
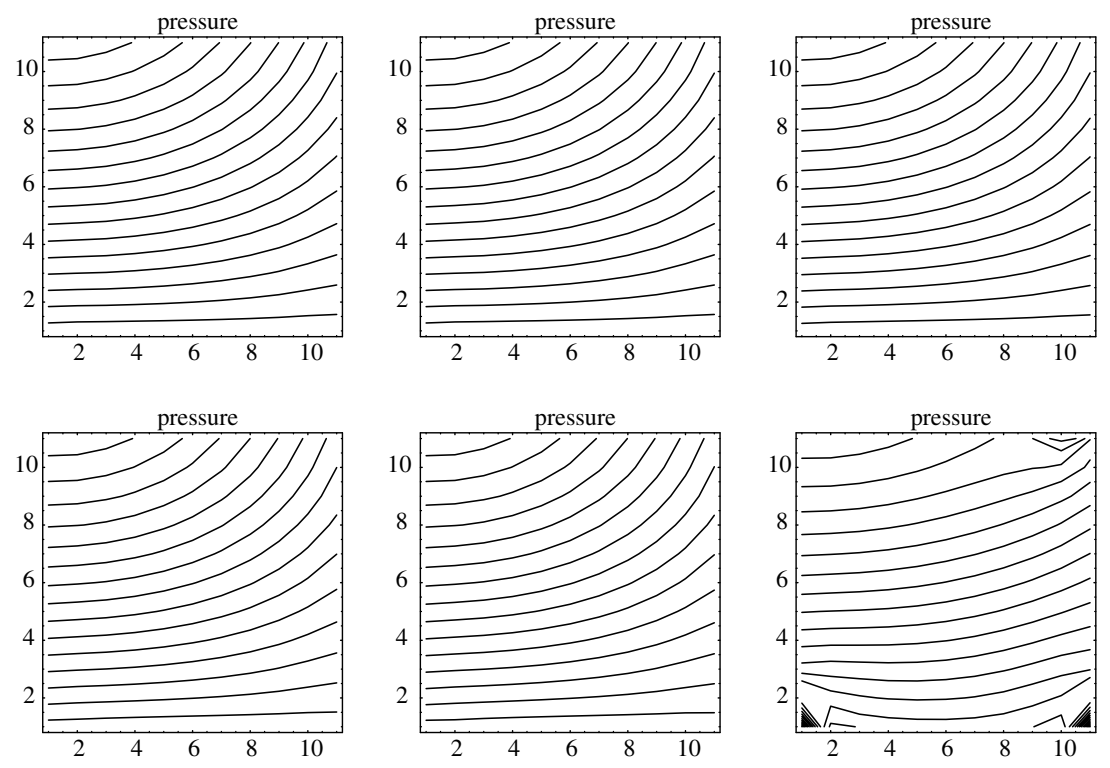

Fig. 1. Pressure approximation for $\rho_{1}, \rho_{2}$ and $\rho_{3}$ (top) and $\rho_{4}, \rho_{5}$ and $\rho_{6}$ (bottom): Taylor-Hood spatial discretization.

As expected, we observe that reduction of the time step has no negative impact on the velocity approximation. The type of $\tau$-definition also does not affect velocity errors which remain virtually identical to the Taylor-Hood errors.

Consider now the pressure approximations. According to (37) the values of $\tau_{\mathrm{S}}$ and $\tau_{\mathrm{T}}$ will be close if $h$ and $\rho$ satisfy (36). In our case $C(\theta)=1, \delta=0.05$ or $\delta=0.04, h \approx 0.1$ and (36) holds if

$$
\rho>\max \{0.05 \times 0.01,0.04 \times 0.01\}=0.0005 .
$$

Inequality (38) is true for $\rho_{1}, \rho_{2}$ and $\rho_{3}$ and we can expect all three methods to yield comparable pressure approximations for these values regardless of the $\tau$ employed. Tables $2-4$ and Figs. 2-7 show that this is indeed the case for the first three time steps.

The remaining three time steps $\rho_{4}, \rho_{5}$ and $\rho_{6}$ do not satisfy (38). We see that violation of this condition also coincides with degradation of the pressure approximation for both $\tau_{\mathrm{T}}$ and $\tau_{\mathrm{S}}$. However, the 
Table 2

Finite element errors after one implicit Euler step: Pressure-Poisson spatial stabilization and P2-P2 elements

\begin{tabular}{|c|c|c|c|c|c|c|c|c|}
\hline \multirow[t]{3}{*}{$n$} & \multicolumn{4}{|c|}{ Velocity $(S)$} & \multicolumn{4}{|c|}{ Pressure $(S)$} \\
\hline & \multicolumn{2}{|c|}{$L^{2}(0.399 \mathrm{E}-3)$} & \multicolumn{2}{|c|}{$H^{1}(0.303 \mathrm{E}-1)$} & \multicolumn{2}{|c|}{$L^{2}(0.450 \mathrm{E}-2)$} & \multicolumn{2}{|c|}{$H^{1}(0.179 \mathrm{E}-1)$} \\
\hline & $\tau_{\mathrm{S}}$ & $\tau_{\mathrm{T}}$ & $\tau_{\mathrm{S}}$ & $\tau_{\mathrm{T}}$ & $\tau_{\mathrm{S}}$ & $\tau_{\mathrm{T}}$ & $\tau_{\mathrm{S}}$ & $\tau_{\mathrm{T}}$ \\
\hline \multicolumn{9}{|l|}{$\rho>0.0005$} \\
\hline 1 & $0.400 \mathrm{E}-3$ & $0.400 \mathrm{E}-3$ & $0.303 \mathrm{E}-1$ & $0.303 \mathrm{E}-1$ & $0.223 \mathrm{E}-2$ & $0.223 \mathrm{E}-2$ & $0.176 \mathrm{E}-1$ & $0.176 \mathrm{E}-1$ \\
\hline 2 & $0.400 \mathrm{E}-3$ & $0.400 \mathrm{E}-3$ & $0.303 \mathrm{E}-1$ & $0.303 \mathrm{E}-1$ & $0.450 \mathrm{E}-2$ & $0.450 \mathrm{E}-2$ & $0.180 \mathrm{E}-1$ & $0.180 \mathrm{E}-1$ \\
\hline 3 & $0.398 \mathrm{E}-3$ & $0.398 \mathrm{E}-3$ & $0.304 \mathrm{E}-1$ & $0.303 \mathrm{E}-1$ & $0.245 \mathrm{E}-1$ & $0.237 \mathrm{E}-1$ & $0.487 \mathrm{E}-1$ & $0.543 \mathrm{E}-1$ \\
\hline \multicolumn{9}{|l|}{$\rho<0.0005$} \\
\hline 4 & $0.398 \mathrm{E}-3$ & $0.393 \mathrm{E}-3$ & $0.304 \mathrm{E}-1$ & $0.304 \mathrm{E}-1$ & $0.141 \mathrm{E}+0$ & $0.332 \mathrm{E}-1$ & $0.295 \mathrm{E}+0$ & $0.151 \mathrm{E}+1$ \\
\hline 5 & $0.398 \mathrm{E}-3$ & $0.391 \mathrm{E}-3$ & $0.304 \mathrm{E}-1$ & $0.305 \mathrm{E}-1$ & $0.446 \mathrm{E}+0$ & $0.784 \mathrm{E}+0$ & $0.113 \mathrm{E}+1$ & $0.213 E+2$ \\
\hline 6 & $0.397 \mathrm{E}-3$ & $0.391 \mathrm{E}-3$ & $0.305 \mathrm{E}-1$ & $0.305 \mathrm{E}-1$ & $0.789 \mathrm{E}+0$ & $0.889 \mathrm{E}+1$ & $0.229 \mathrm{E}+1$ & $0.221 E+3$ \\
\hline
\end{tabular}

Table 3

Finite element errors after one implicit Euler step: Douglas-Wang spatial stabilization and P2-P2 elements

\begin{tabular}{|c|c|c|c|c|c|c|c|c|}
\hline \multirow[t]{3}{*}{$n$} & \multicolumn{4}{|c|}{ Velocity $(S)$} & \multicolumn{4}{|c|}{ Pressure $(S)$} \\
\hline & \multicolumn{2}{|c|}{$L^{2}(0.399 \mathrm{E}-3)$} & \multicolumn{2}{|c|}{$H^{1}(0.304 \mathrm{E}-1)$} & \multicolumn{2}{|c|}{$L^{2}(0.228 \mathrm{E}-2)$} & \multicolumn{2}{|c|}{$H^{1}(0.157 \mathrm{E}-1)$} \\
\hline & $\tau_{\mathrm{S}}$ & $\tau_{\mathrm{T}}$ & $\tau_{\mathrm{S}}$ & $\tau_{\mathrm{T}}$ & $\tau_{\mathrm{S}}$ & $\tau_{\mathrm{T}}$ & $\tau_{\mathrm{S}}$ & $\tau_{\mathrm{T}}$ \\
\hline \multicolumn{9}{|c|}{$\rho>0.0005$} \\
\hline 1 & $0.398 \mathrm{E}-3$ & $0.398 \mathrm{E}-3$ & $0.304 \mathrm{E}-1$ & $0.304 \mathrm{E}-1$ & $0.241 \mathrm{E}-2$ & $0.241 \mathrm{E}-2$ & $0.157 \mathrm{E}-1$ & $0.157 \mathrm{E}-1$ \\
\hline 2 & $0.398 \mathrm{E}-3$ & $0.398 \mathrm{E}-3$ & $0.304 \mathrm{E}-1$ & $0.304 \mathrm{E}-1$ & $0.356 \mathrm{E}-2$ & $0.356 \mathrm{E}-2$ & $0.164 \mathrm{E}-1$ & $0.164 \mathrm{E}-1$ \\
\hline 3 & $0.398 \mathrm{E}-3$ & $0.398 \mathrm{E}-3$ & $0.304 \mathrm{E}-1$ & $0.304 \mathrm{E}-1$ & $0.145 \mathrm{E}-1$ & $0.142 \mathrm{E}-1$ & $0.350 \mathrm{E}-1$ & $0.438 \mathrm{E}-1$ \\
\hline \multicolumn{9}{|c|}{$\rho<0.0005$} \\
\hline 4 & $0.398 \mathrm{E}-3$ & $0.393 \mathrm{E}-3$ & $0.304 \mathrm{E}-1$ & $0.304 \mathrm{E}-1$ & $0.999 \mathrm{E}-1$ & $0.299 \mathrm{E}-1$ & $0.235 \mathrm{E}+0$ & $0.151 \mathrm{E}+1$ \\
\hline 5 & $0.398 \mathrm{E}-3$ & $0.391 \mathrm{E}-3$ & $0.304 \mathrm{E}-1$ & $0.305 \mathrm{E}-1$ & $0.433 \mathrm{E}+0$ & $0.783 \mathrm{E}+0$ & $0.114 \mathrm{E}+1$ & $0.213 E+2$ \\
\hline 6 & $0.397 \mathrm{E}-3$ & $0.391 \mathrm{E}-3$ & $0.304 \mathrm{E}-1$ & $0.305 \mathrm{E}-1$ & $0.852 \mathrm{E}+0$ & $0.890 \mathrm{E}+1$ & $0.246 \mathrm{E}+1$ & $0.221 \mathrm{E}+3$ \\
\hline
\end{tabular}

Table 4

Finite element errors after one implicit Euler step: Galerkin-Least-Squares spatial stabilization and P2-P2 elements

\begin{tabular}{|c|c|c|c|c|c|c|c|c|}
\hline \multirow[t]{3}{*}{$n$} & \multicolumn{4}{|c|}{ Velocity $(S)$} & \multicolumn{4}{|c|}{ Pressure $(S)$} \\
\hline & \multicolumn{2}{|c|}{$L^{2}(0.713 \mathrm{E}-3)$} & \multicolumn{2}{|c|}{$H^{1}(0.438 \mathrm{E}-1)$} & \multicolumn{2}{|c|}{$L^{2}(0.237 \mathrm{E}-2)$} & \multicolumn{2}{|c|}{$H^{1}(0.176 \mathrm{E}-1)$} \\
\hline & $\tau_{\mathrm{S}}$ & $\tau_{\mathrm{T}}$ & $\tau_{\mathrm{S}}$ & $\tau_{\mathrm{T}}$ & $\tau_{\mathrm{S}}$ & $\tau_{\mathrm{T}}$ & $\tau_{\mathrm{S}}$ & $\tau_{\mathrm{T}}$ \\
\hline \multicolumn{9}{|c|}{$\rho>0.0005$} \\
\hline 1 & $0.690 \mathrm{E}-3$ & $0.690 \mathrm{E}-3$ & $0.435 \mathrm{E}-1$ & $0.435 \mathrm{E}-1$ & $0.319 \mathrm{E}-2$ & $0.319 \mathrm{E}-2$ & $0.175 \mathrm{E}+0$ & $0.175 \mathrm{E}+0$ \\
\hline 2 & $0.601 \mathrm{E}-3$ & $0.600 \mathrm{E}-3$ & $0.408 \mathrm{E}-1$ & $0.408 \mathrm{E}-1$ & $0.209 \mathrm{E}-1$ & $0.208 \mathrm{E}-1$ & $0.171 \mathrm{E}+0$ & $0.171 \mathrm{E}+0$ \\
\hline 3 & $0.438 \mathrm{E}-3$ & $0.430 \mathrm{E}-3$ & $0.326 \mathrm{E}-1$ & $0.322 \mathrm{E}-1$ & $0.909 \mathrm{E}-1$ & $0.820 \mathrm{E}-1$ & $0.248 \mathrm{E}+0$ & $0.255 \mathrm{E}+0$ \\
\hline \multicolumn{9}{|c|}{$\rho<0.0005$} \\
\hline 4 & $0.396 \mathrm{E}-3$ & $0.392 \mathrm{E}-3$ & $0.304 \mathrm{E}-1$ & $0.304 \mathrm{E}-1$ & $0.175 \mathrm{E}+0$ & $0.364 \mathrm{E}-1$ & $0.431 \mathrm{E}+0$ & $0.160 \mathrm{E}+1$ \\
\hline 5 & $0.397 \mathrm{E}-3$ & $0.391 \mathrm{E}-3$ & $0.304 \mathrm{E}-1$ & $0.305 \mathrm{E}-1$ & $0.330 \mathrm{E}+0$ & $0.786 \mathrm{E}+0$ & $0.899 \mathrm{E}+0$ & $0.213 E+2$ \\
\hline 6 & $0.397 \mathrm{E}-3$ & $0.391 \mathrm{E}-3$ & $0.304 \mathrm{E}-1$ & $0.305 \mathrm{E}-1$ & $0.547 \mathrm{E}+0$ & $0.890 \mathrm{E}+1$ & $0.173 \mathrm{E}+1$ & $0.221 E+3$ \\
\hline
\end{tabular}

manifestation of this deterioration is different for the different $\tau$-definitions. Recall that for small $\rho$ a stabilized method that employs $\tau_{\mathrm{T}}$ reduces to a mixed formulation. The expected outcome is a pressure field polluted by spurious oscillations. This is exactly what we see in Figs. 5-7. 

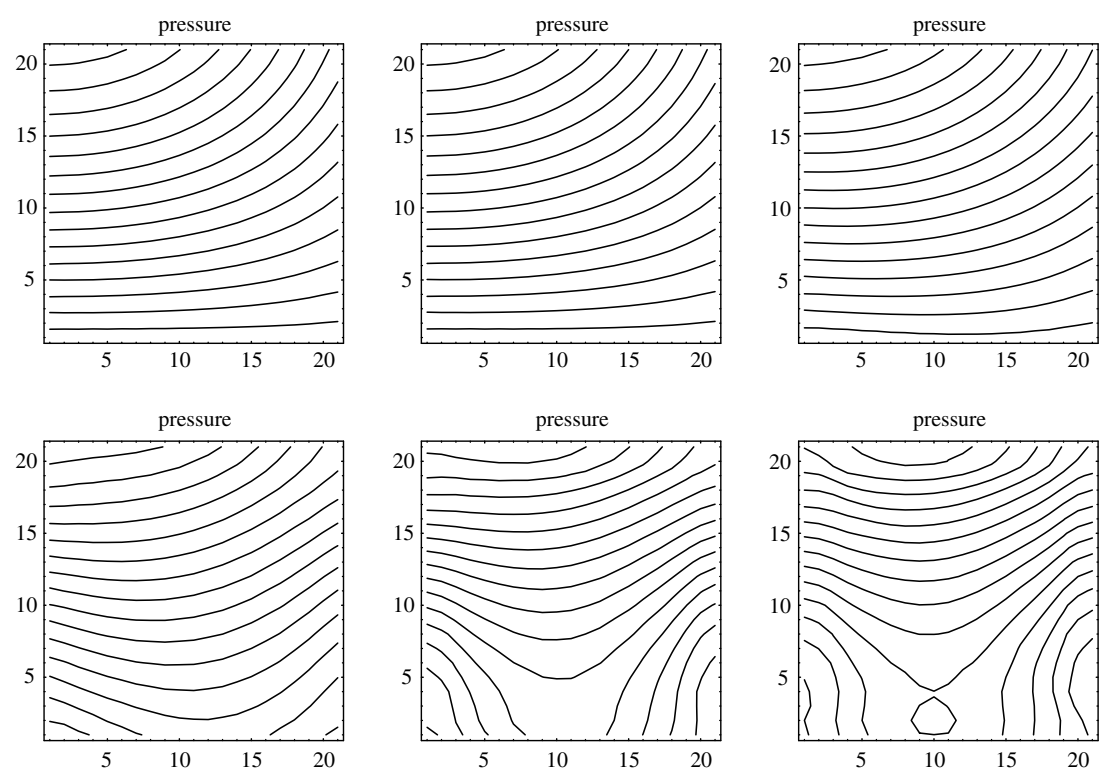

Fig. 2. Pressure approximation for $\rho_{1}, \rho_{2}$ and $\rho_{3}$ (top) and $\rho_{4}, \rho_{5}$ and $\rho_{6}$ (bottom): Pressure-Poisson stabilization with $\tau_{\mathrm{S}}$.
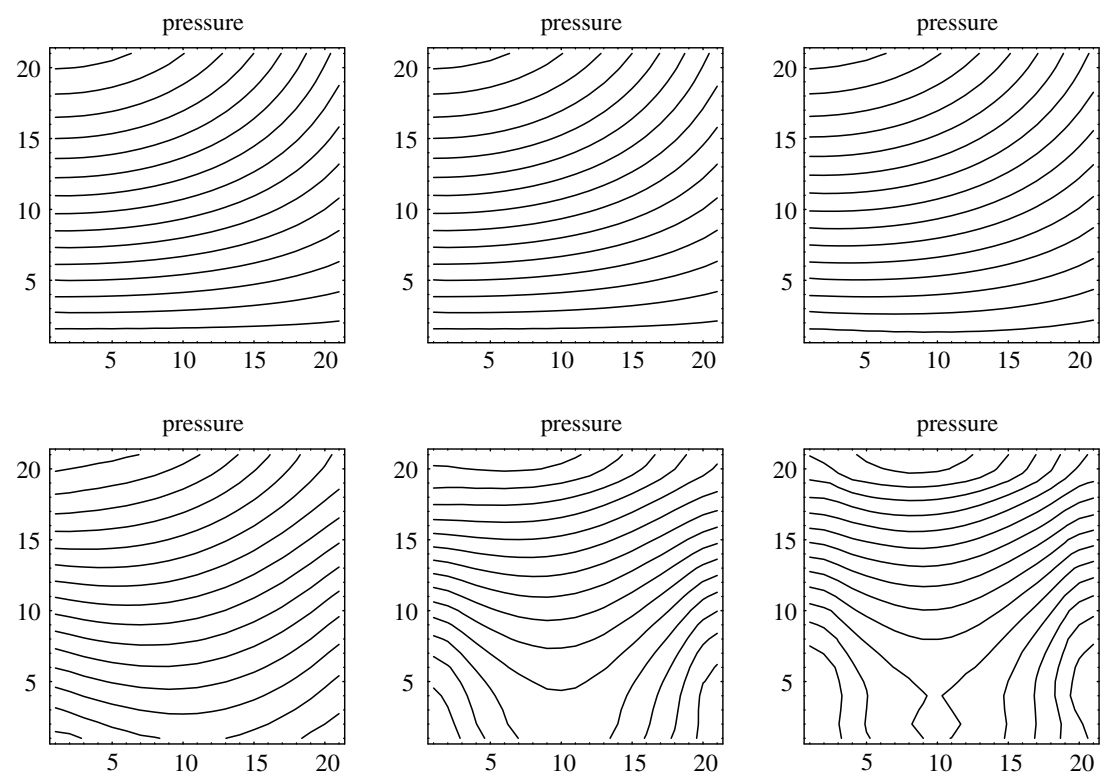

Fig. 3. Pressure approximation for $\rho_{1}, \rho_{2}$ and $\rho_{3}$ (top) and $\rho_{4}, \rho_{5}$ and $\rho_{6}$ (bottom): Douglas-Wang stabilization with $\tau_{\mathrm{S}}$.

In contrast, when $\tau_{\mathrm{S}}$ is employed, pressure approximations do not develop significant oscillations even for the smallest time step. However, as the time step is being reduced, pressure begins to deviate from the expected profile. For Pressure-Poisson and Douglas-Wang methods deviation occurs at $\rho_{4}$, for the 

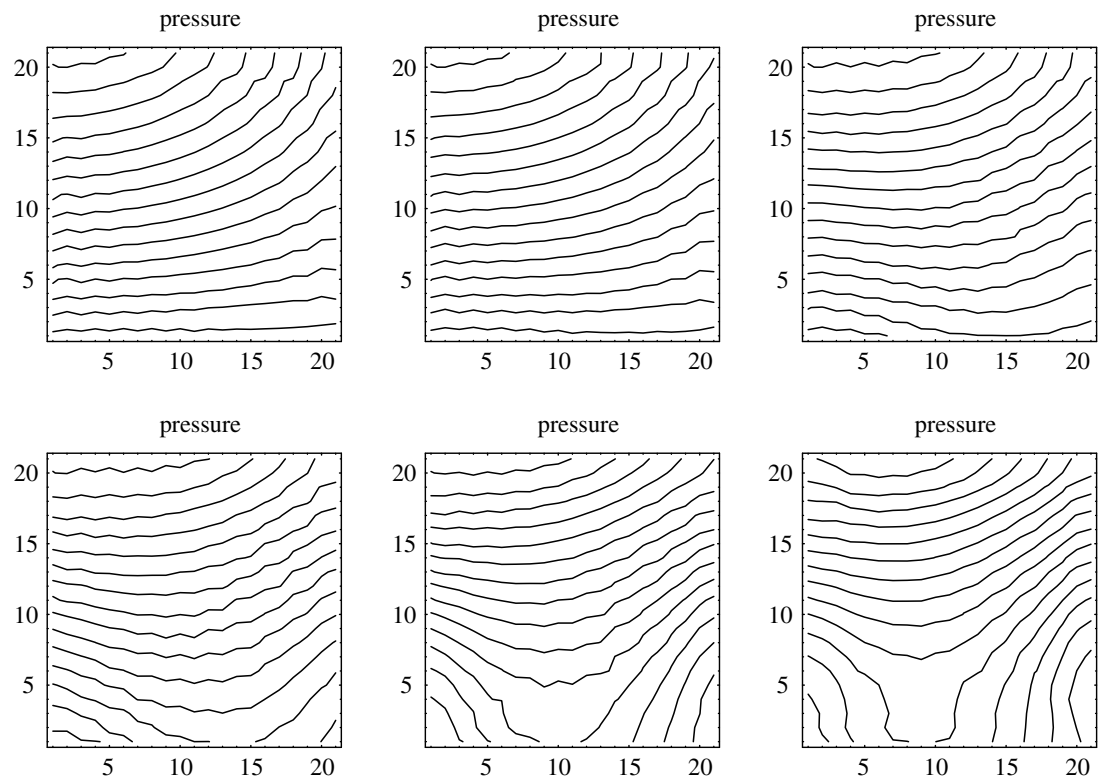

Fig. 4. Pressure approximation for $\rho_{1}, \rho_{2}$ and $\rho_{3}$ (top) and $\rho_{4}, \rho_{5}$ and $\rho_{6}$ (bottom): Galerkin-Least-Squares stabilization with $\tau_{\mathrm{S}}$.
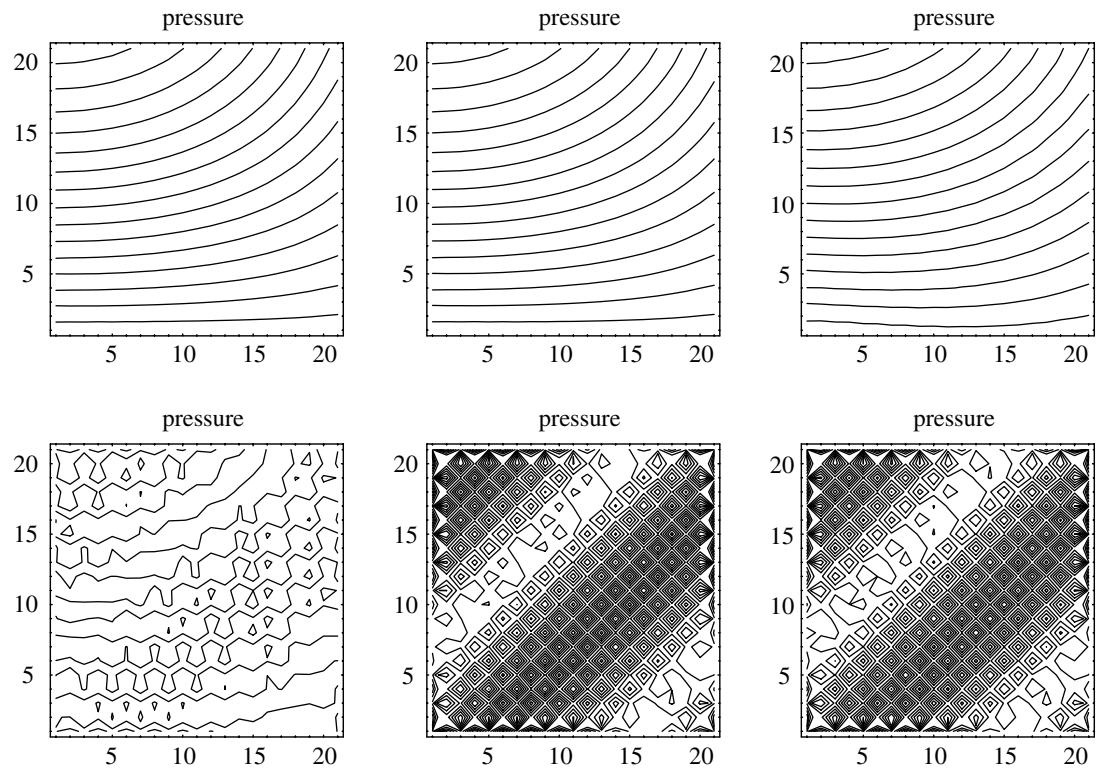

Fig. 5. Pressure approximation for $\rho_{1}, \rho_{2}$ and $\rho_{3}$ (top) and $\rho_{4}, \rho_{5}$ and $\rho_{6}$ (bottom): Pressure-Poisson stabilization with $\tau_{\mathrm{T}}$.

Galerkin-Least-Squares it occurs earlier at $\rho_{3}$. These values are in a very good agreement with the sufficient bound in (36). 

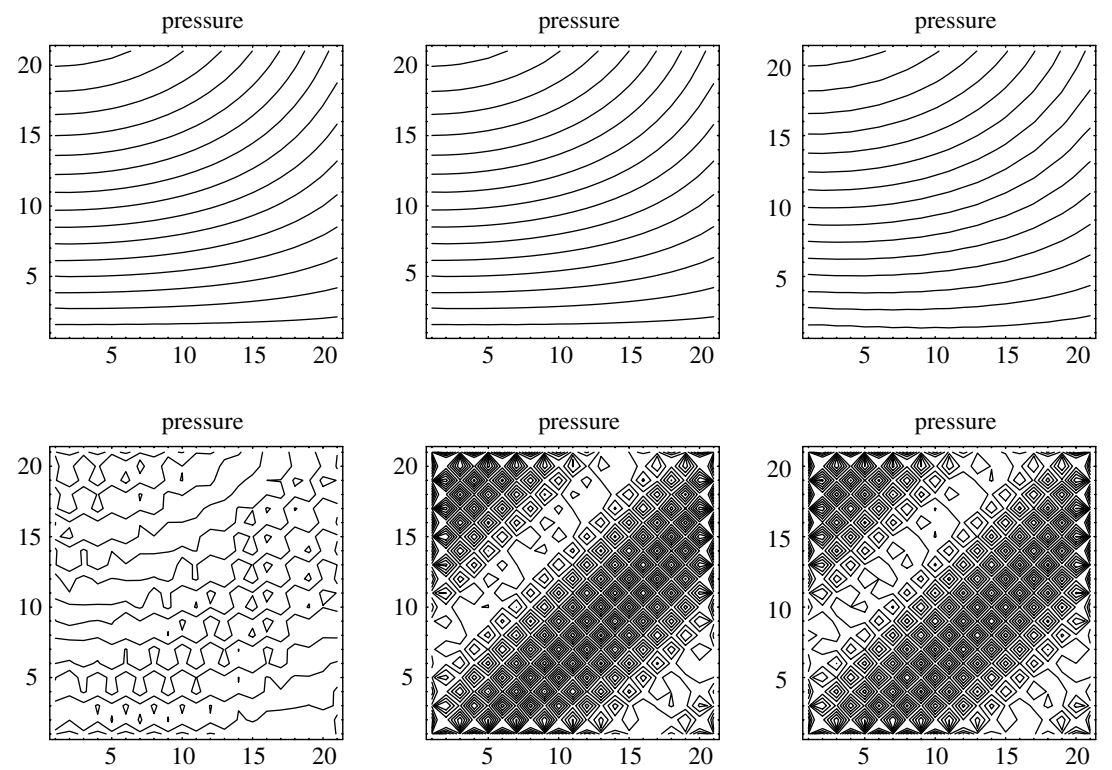

Fig. 6. Pressure approximation for $\rho_{1}, \rho_{2}$ and $\rho_{3}$ (top) and $\rho_{4}, \rho_{5}$ and $\rho_{6}$ (bottom): Douglas-Wang stabilization with $\tau_{\mathrm{T}}$.
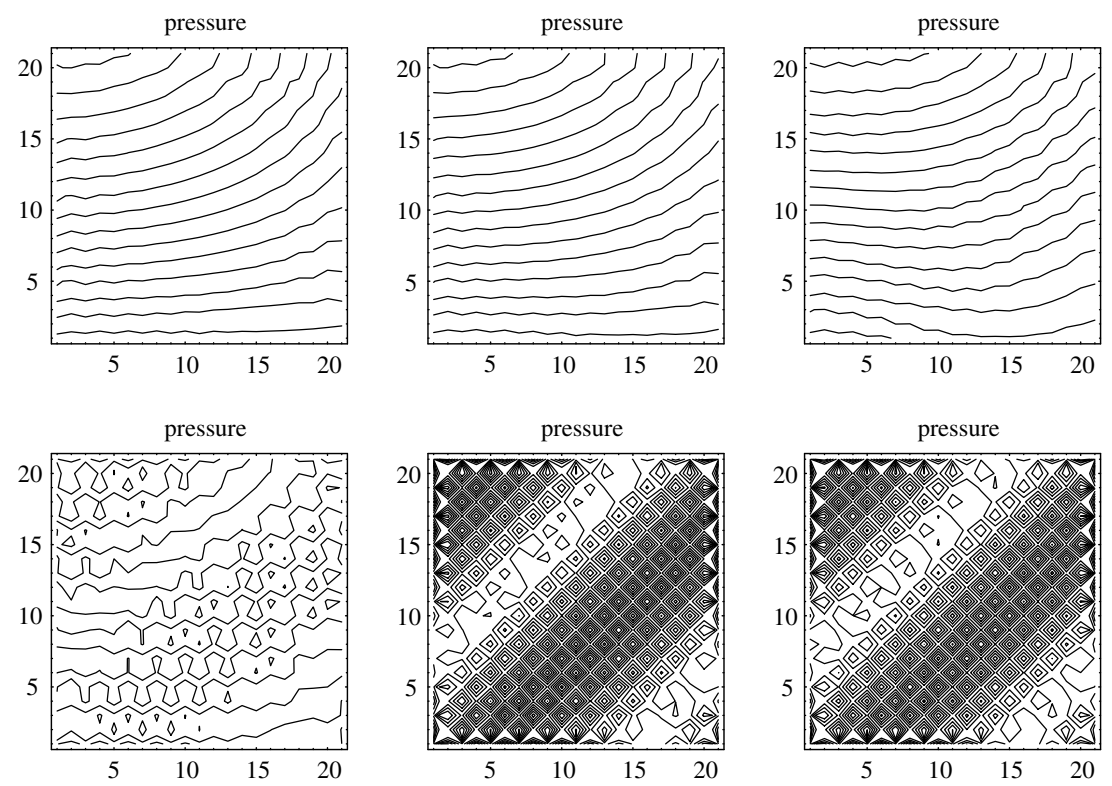

Fig. 7. Pressure approximation for $\rho_{1}, \rho_{2}$ and $\rho_{3}$ (top) and $\rho_{4}, \rho_{5}$ and $\rho_{6}$ (bottom): Galerkin-Least-Squares stabilization with $\tau_{\mathrm{T}}$.

To summarize, our experiments indicate that

$$
h^{2}<C \frac{\rho}{\delta}
$$


where $C$ is a constant that depends on the type of implicit time scheme but not on the values of $h$ and $\rho$, can be used as a reliable indicator for the threshold values of the time step beyond which methods will experience problems with stability or accuracy.

\section{Conclusions}

We have demonstrated theoretically and numerically that implicit time integration combined with infsup stabilized spatial discretization may lead to anomalous solutions for very small time steps and fixed spatial mesh. The type of anomalies depends on the type of $\tau$-definition employed in the method. For transient stability parameters small time steps lead to spurious pressure oscillations while for spatial stability parameters pressure profiles begin to deviate from the exact solution. In both cases the onset of anomalous behavior occurs when time step becomes smaller than the threshold value $\rho \approx C(\theta) \delta h^{2}$. As a result, the spatial grid size cannot be chosen independently from the time step, if stable and accurate approximation is desired. For problems with multiple time scales this stability prerequisite forces an excessive, non-physical spatial refinement and significant increase in computational cost.

Since the cause for the anomalous behavior is the coupling between the spatial and temporal scales engendered by the "mass" term (20), problems can be avoided by either lagging or completely discarding this term. This effectively renders the scheme into a penalty-like formulation. Alternatives that can both maintain consistency and provide stability are to employ time-space elements or to use stable pairs of finite element spaces. Further studies are also needed to reveal the source of the anomalous pressure behavior when the spatial stability parameter $\tau_{\mathrm{S}}$ is employed in the methods. These will be reported in a forthcoming paper.

\section{Acknowledgements}

We would like to thank Marek Behr, Isaac Harari and Rich Lehoucq for helpful discussions. We also thank the anonymous referees for the helpful comments and suggestions.

\section{References}

[1] T. Barth, P. Bochev, M. Gunzburger, J. Shadid, A taxonomy of consistently stabilized finite element methods for the Stokes problem, SIAM J. Sci. Comput., submitted for publication.

[2] M. Behr, Stabilized Finite Element Methods for Incompressible Flows with Emphasis on Moving Boundaries and Interfaces, Ph.D. thesis, University of Minnesota, Department of Aerospace Engineering and Mechanics, 1992.

[3] M. Behr, Personal communication, 2001.

[4] M.A. Behr, L.P. Franca, T.E. Tezduyar, Stabilized finite element methods for the velocity-pressure-stress formulation of incompressible flows, Comput. Methods Appl. Mech. Engrg. 104 (1993) 31-48.

[5] J. Blasco, R. Codina, Stabilized finite element method for the transient Navier-Stokes equations based on a pressure gradient projection, Comput. Methods Appl. Mech. Engrg. 182 (2000) 277-300.

[6] J. Blasco, R. Codina, Space and time error estimates for a first-order, pressure stabilized finite element method for the incompressible Navier-Stokes equations, Appl. Numer. Math. 38 (2001) 475-497.

[7] P. Bochev, M. Gunzburger, J. Shadid, On stabilized finite element methods for transient problems with varying time scales, in: J. Eberhardsteiner, H. Mang, F. Rammerstorfer (Eds.), Proceedings of the Fifth World Congress on Computational Mechanics, Vienna, Austria, 7-12 July 2002, TU Vienna, Technical University, Vienna.

[8] S.F. Bradford, N.D. Katopodes, The antidissipative, non-monotone behavior of Petrov-Galerkin upwinding, Int. J. Numer. Methods Fluids 33 (2000) 583-608.

[9] F. Brezzi, On existence, uniqueness and approximation of saddle-point problems arising from Lagrange multipliers, RAIRO Model. Math. Anal. Numer. 21 (1974) 129-151. 
[10] F. Brezzi, J. Douglas, Stabilized mixed methods for the Stokes problem, Numer. Math. 53 (1988) 225-235.

[11] C. Johnson, U. Navert, J. Pitkaranta, Finite element methods for linear hyperbolic problems, Comput. Methods Appl. Mech. Engrg. 45 (1984) 285-312.

[12] J. Douglas, J. Wang, An absolutely stabilized finite element method for the Stokes problem, Math. Comput. (1989) $52-495$.

[13] L. Franca, S. Frey, T. Hughes, Stabilized finite element methods: I. Application to the advective-diffusive model, Comput. Methods Appl. Mech. Engrg. 95 (1992) 253-276.

[14] L. Franca, S. Frey, Stabilized finite element methods: II. The incompressible Navier-Stokes equations, Comput. Methods Appl. Mech. Engrg. 99 (1991) 209-233.

[15] L.P. Franca, E.G. Dutra do Carmo, The Galerkin gradient least-squares method, Comput. Methods Appl. Mech. Engrg. 74 (1989) 41-54.

[16] L.P. Franca, C. Farhat, Bubble functions prompt unusual stabilized finite element methods, Comput. Methods Appl. Mech. Engrg. 123 (1995) 299-308.

[17] L.P. Franca, F. Valentin, On an improved unusual stabilized finite element method for advective-reactive-diffusive equations, Comput. Methods Appl. Mech. Engrg. 189 (2000) 1785-1800.

[18] I. Harari, Spatial stability of semidiscrete formulations for parabolic problems, in: J. Eberhardsteiner, H. Mang, F. Rammerstorfer (Eds.), Proceedings of the Fifth World Congress on Computational Mechnics, Vienna, Austria, 7-12 July 2002, TU Vienna, Technical University, Vienna.

[19] I. Harari, Stability of semidiscrete formulations for parabolic problems at small time steps, Comput. Methods Appl. Mech. Engrg., in press.

[20] T.J.R. Hughes, Multiscale phenomena: Green's function, the Dirichlet-to-Neumann map, subgrid scale models, bubbles and the origins of stabilized methods, Comput. Methods Appl. Mech. Engrg. 127 (1995) 387-401.

[21] T.J.R. Hughes, A. Brooks, A theoretical framework for Petrov-Galerkin methods with discontinuous weighting functions: Application to the streamline-upwind procedure, in: R.H. Gallagher et al. (Eds.), Finite Elements in Fluids, vol. 4, J. Willey \& Sons, 1982, pp. 47-65.

[22] T.J.R. Hughes, G.R. Feijoo, L. Mazzei, J.B. Quincy, The variational multiscale method: A paradigm for computational mechanics, Comput. Methods Appl. Mech. Engrg. 166 (1998) 3-24.

[23] T.J.R. Hughes, L. Franca, A new finite element formulation for computational fluid dynamics: VII. The Stokes problem with various well-posed boundary conditions: symmetric formulations that converge for all velocity pressure spaces, Comput. Methods Appl. Mech. Engrg. 65 (1987) 85-96.

[24] T.J.R. Hughes, L. Franca, M. Balestra, A new finite element formulation for computational fluid dynamics: V. Circumventing the Babuska-Brezzi condition: A stable Petrov-Galerkin formulation of the Stokes problem accommodating equal-order interpolations, Comput. Methods Appl. Mech. Engrg. 59 (1986) 85-99.

[25] T.J.R. Hughes, M. Mallet, A. Mizukami, A new finite element formulation for computational fluid dynamics: II. Beyond SUPG, Comput. Methods Appl. Mech. Engrg. 54 (1986) 341-355.

[26] F. Ilinica, J.-F. Hetu, Galerkin gradient least-squares formulations for transient conduction heat transfer, Comput. Methods Appl. Mech. Engrg. 191 (2002) 3073-3097.

[27] P. Ciarlet, Finite Element Method for Elliptic Problems, North Holland, Amsterdam, 1978.

[28] J.N. Shadid, C. Ober, Preliminary studies of time-discretization accuracy with MP Salsa, 2002, unpublished.

[29] F. Shakib, Finite Element Analysis of the Compressible Euler and Navier-Stokes Equations, PhD thesis, Division of Applied Mathematics, Stanford University, 1989.

[30] F. Shakib, T.J.R. Hughes, A new finite element formulation for computational fluid dynamics: IX. fourier analysis of space-time Galerkin/least-squares algorithms, Comput. Methods Appl. Mech. Engrg. 87 (1991) 35-58.

[31] F. Shakib, T.J.R. Hughes, Z. Johan, A new finite element formulation for computational fluid dynamics: X. the compressible Euler and Navier-Stokes equations, Comput. Methods Appl. Mech. Engrg. 89 (1991) 141-219.

[32] T. Tezduyar, Y. Osawa, Finite element stabilization parameters computed from element matrices and vectors, Comput. Methods Appl. Mech. Engrg. 190 (2000) 411-430. 